\title{
A new 4-D hyperchaotic hidden attractor system: Its dynamics, coexisting attractors, synchronization and microcontroller implementation
}

\author{
Basil H. Jasim ${ }^{1}$, Kadhim H. Hassan ${ }^{2}$, Khulood Moosa Omran ${ }^{3}$ \\ ${ }^{1}$ Electrical Engineering Department, College of Engineering, University of Basrah, Iraq \\ ${ }^{2}$ Control and automation Department, Basrah Engineering Technical College, Southern Technical University, Iraq \\ ${ }^{3}$ Electrical Engineering Department, College of Engineering, University of Basrah, Iraq
}

\begin{tabular}{l} 
Article Info \\
Article history: \\
Received Jul 8, 2020 \\
Revised Sep 22, 2020 \\
Accepted Sep 30, 2020 \\
\hline
\end{tabular}

Keywords:

Chaotic

Coexisting attractors

Hidden attractor

Hyperchaotic

Synchronization

\begin{abstract}
In this paper, a simple 4-dimensional hyperchaotic system is introduced. The proposed system has no equilibria points, so it admits hidden attractor which is an interesting feature of chaotic systems. Another interesting feature of the proposed system is the coexisting of attractors where it shows periodic and chaotic coexisting attractors. After introducing the system, the system is analyzed dynamically using numerical and theoretical techniques. In this analysis, Lyapunov exponents and bifurcation diagrams have been used to investigate chaotic and hyperchaotic nature, the ranges of system parameters for different behaviors and the route for chaos and coexisting attractors regions. In the next part of our work, a synchronization control system for two identical systems is designed. The design procedure uses a combination of simple synergetic control with adaptive updating laws to identify the unknown parameters derived basing on Lyapunov theorem. Microcontroller (MCU) based hardware implementation system is proposed and tested by using MATLAB as a display side. As an application, the designed synchronization system is used as a secure analog communication system. The designed MCU system with MATLAB Simulation is used to validate the designed synchronization and secure communication systems and excellent results have been obtained.
\end{abstract}

This is an open access article under the CC BY-SA license.

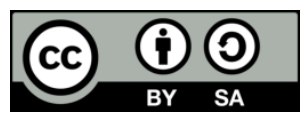

\section{Corresponding Author:}

Basil H. Jasim

Electrical Engineering Department, College of Engineering

University of Basrah, Iraq

Email: basil.jasim@uobasrah.edu.iq

\section{INTRODUCTION}

The dynamical system which highly effected by initial condition is known as chaotic system. Chaotic systems are characterized by their strange attractors. Due to their attraction, theoretical importance and possible scientific and engineering applications, chaotic systems received great attention in the literature and scientific research [1]. Analytically, there are several methods to investigate the existence of chaotic behavior in dynamical systems, the most important of these methods are Lyapunov exponents and bifurcation diagrams [2]. Dynamical systems having positive maximum Lyapunov exponent are chaotic [3]. The largest maximum positive exponent system is the more chaos one. Hyperchaotic system is a chaotic system with more than one positive Lyapunov exponents $[4,5]$. Hyperchaotic systems show more dynamical complexity comparing with that systems which have one Lyapunov exponent or simply ordinary chaotic systems [6]. Chaotic systems get great attention in last decades due to their theoretical and practical applications and in 
various fields such as in secure communication, neural networks, laser, nonlinear circuits, mobile robots, oscillators, artificial neural networks, chemical reactors, finance systems, circuits and others [7-11]. According to some researchers, chaos can be regarded as the third largest scientific revolution in the past and current century $[12,13]$.

Since the first arise of chaotic systems by Lorenz [14], several chaotic and hyperchaotic systems with ordinary differential equations, complex and fractional order have been introduced and extensively studied [4, 7, 9, 15-17]. Despite of this fact, but it is still advantageous for the field of chaotic in theoretical and practical areas to find and analyze new chaotic systems [17-18]. Due to their dynamical complexity over chaotic systems, hyperchaotic systems admits more benefits in some applications, especially in the secure communication where it has been shown that encryption with hyperchaotic system gives more efficient secure communication system [19].

In classical chaotic theory, the excitation of strange attractors of chaotic systems comes from their unstable equilibria, so they are referred to as self-excited chaotic systems [8,9]. More recently, another type of chaotic systems has been appeared, where no equilibria or just a stable equilibrium points system with chaotic behavior $[20,21]$. These types of systems have been known as hidden attractors chaos and the reason for this name is very clear. In chaotic literature, it can easily be noted that hyperchaotic with hidden attractor systems are considerably interesting systems [22]. Coexisting attractors is an interesting feature which rarely appear in systems and it means that the system shows different motions for different initial conditions (IC).

Synchronization of two or more chaotic systems received considerable attention due to its theoretical and practical importance in several fields such as secure communication [23, 24]. Sliding mode control theory, adaptive control, active control and other approaches have been used for solving the challenges of the problem of chaotic systems synchronization [25]. Multi wings attractors is another feature of chaotic system where the phase portraits of the chaotic system displays more than on part which are usually identical and this feature has appeared early in Lorenz work [14]. Recently, many research articles have focused on this feature and as example the works in [26, 27].

Motivated by the previous discussion, we present in this work, a new system which have the three interesting features of chaos systems and namely it is hyperchaotic with hidden attractor system and gives coexisting attractors feature. An extensive study including the important dynamical properties of the proposed system is presented. An efficient MCU implementation is presented and synchronization controllers are designed and finally an application of the synchronization system is proposed and tested experimentally and by simulation study.

The rest of the paper is organized as follows: in section 2, the new system is introduced. The dynamical system analysis and its properties are presented in section 3. A tracking control system design for the system is presented in section 4. In section 5, a synchronization control system is designed. In section 6, simulation study is presented and used to validate the effectiveness of the control and synchronization system and to use the designed synchronization system to build and test a secure communication system. Finally, in section 7, paper conclusion has been drawn.

\section{THE NEW SYSTEM}

The following system of equations which have two quadratic nonlinearity terms represents the new system:

$$
\begin{aligned}
& \dot{x}_{1}=a x_{3} \\
& \dot{x}_{2}=x_{3} x_{4}-b \\
& \dot{x}_{3}=x_{1}-x_{2} x_{4} \\
& \dot{x}_{4}=x_{3}-x_{4}
\end{aligned}
$$

In this system, a and $\mathrm{b}$ are two positive real parameters. The system is really simple where just two nonlinearities are presented which is the minimum number of nonlinearities required for chaotic behavior, to our knowledge. To obtain the equilibrium points of a system, the left side of the system equations should be equated to zero:

$$
\begin{aligned}
& \mathrm{ax}_{3}=0 \\
& \mathrm{x}_{3} \mathrm{x}_{4}-\mathrm{b}=0 \\
& \mathrm{x}_{1}-\mathrm{x}_{2} \mathrm{x}_{4}=0 \\
& \mathrm{x}_{3}-\mathrm{x}_{4}=0
\end{aligned}
$$


Then, solving for the states of the system (the $X=\left\{\mathrm{x}_{1}, \mathrm{x}_{2}, \mathrm{x}_{3}, \mathrm{x}_{4}\right\}$ vector). It is easily noted that our system has no any solution because from 2.1 and 2.4 the states $\mathrm{x}_{3}$ and $\mathrm{x}_{4}$ equal to zero and this result cannot be consistent with 2.2 for non-zero values of $b$. Then, there are no solution, and hence there are no equilibrium points for this system and any strange attractor generated by the system is a hidden attractor.

System 1 shows chaotic behaviors for different values of parameters ( $a$ and $b$ ). As instant for $a=3.5$ and $b=0.05$, the phase portraits clearly show the chaotic nature of the system. Figures 1 and 2 , show the 2 dimensional hyperchaotic attractors of the system for $\mathrm{x}_{1}, \mathrm{x}_{2}$ and $\mathrm{x}_{2}, \mathrm{x}_{3}$ with $\{0.1,0.1,0.1,0.1\}$ as an initial conditions and $500 \mathrm{sec}$. simulation time.

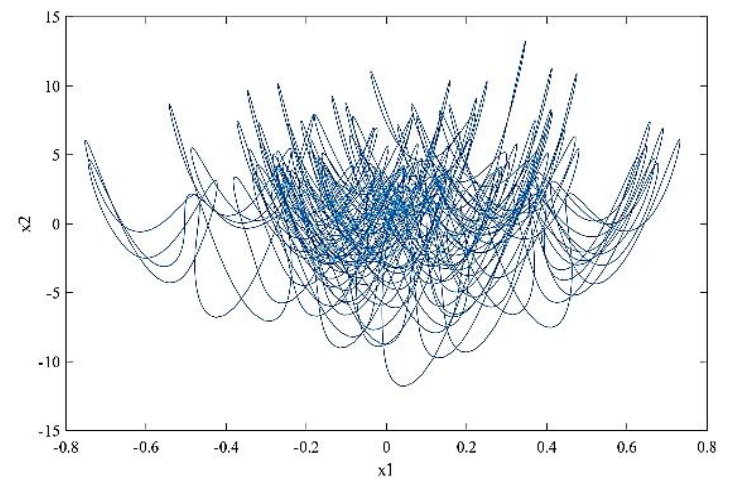

Figure 1. 2 D phase portrait of $\mathrm{x}_{1}, \mathrm{x}_{2}$

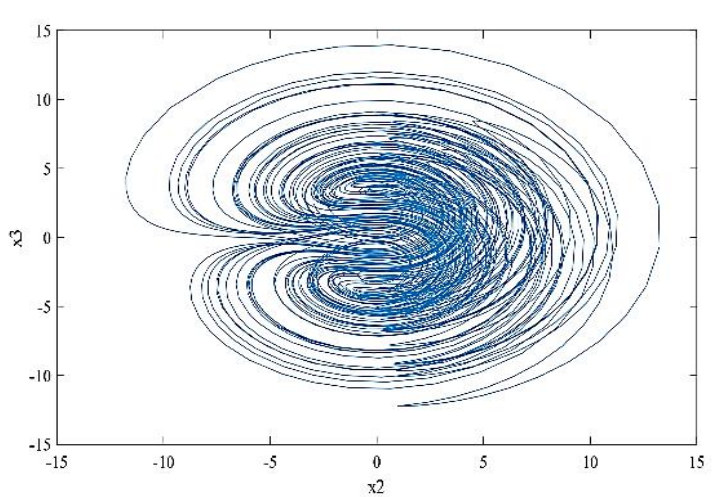

Figure 2. 2 D phase portrait of $\mathrm{x}_{2}, \mathrm{x}_{3}$

\subsection{Symmetry}

It can easily be noted that the system is invariant under the transformation:

$$
\left(x_{1}, x_{2}, x_{3}, x_{4}\right) \rightarrow\left(-x_{1}, x_{2},-x_{3},-x_{4}\right)
$$

which means that if $x_{1}, x_{3}, x_{1}, x_{3}$ are a solution for the system equations, then $-x_{1}, x_{2},-x_{3},-x_{4}$ is also a solution for it.

\subsection{Dissipation}

To investigate whether the system is dissipative or not, the divergence of the system should be calculated. To this end, the system is rewritten as a vector notation:

$$
V(X)=\left[\begin{array}{l}
v_{1} \\
v_{2} \\
v_{3} \\
v_{4}
\end{array}\right]=\left[\begin{array}{c}
a x_{3} \\
x_{3} x_{4}-b \\
x_{1}-x_{2} x_{4} \\
x_{3}-x_{4}
\end{array}\right]
$$

Then, the divergence of the system can be obtained by the following:

$$
\nabla \cdot f=\sum_{i=1}^{4} \frac{\partial v_{i}}{\partial x_{i}}=0+0+0-1=-1<0
$$

The negative sign of the divergence value indicates that the system is dissipative. System dissipation means that the system will settle to a set of zero measurement for any asymptotic motion. Which leads to chaotic behavior.

\section{SYSTEM ANALYSIS}

\subsection{System analysis using Lyapunov exponents}

System in (1) shows chaotic behavior for certain sets of values of a and b. To investigate these values and determining system behavior over the full range of $a$ and $b$, and also to determine whether the 
system can exhibit hyperchaotic attractor, we used first and second Lyapunov exponents. We selected a $=0.02$ as a rough value from its chaotic range, then the Lyapunov exponents for $b=\left[\begin{array}{ll}1 & 10\end{array}\right]$ with a step equal to 0.04 has been calculated using $\{0.1,0.1,0.1,0.1\}$ as an initial condition. Lyapunov exponents have been found by using Alen wolf algorithm [28]. The system has been simulated using Runge-Kuta method for each value of $\mathrm{b}$ and for sufficient time $(1000 \mathrm{sec}$.) to reach to exponents settling values. MATLAB 2018a has been used in this analysis. Figure 3 shows the four Lyapunov exponents with $\mathrm{b}$ changing. Figure 4 and Figure 5 shows the maximum and the second Lyapunov exponents with $b$ changing. These figures show that the system is chaotic for $b=[2.710]$, and within this range of $b$ the system is hyperchaotic for some subranges. Also, the same process has been repeated for $a$ where $b$ has been fixed to 3.5 and a has been changed from 0 to 0.2 with 0.001 step size using the same IC. Figure 6 shows the resulting plot, and from this figure it is noted that the system is hyperchaotic from 0 to 0.085 . As a specific case, we put $a=3.5$ and $b=0.05$ and finding Lyapunov exponents for 2000 secs. simulation time, the settling values for Lyapunov exponents in this case are:

$$
L_{1}=0.3, \quad L_{2}=0.0024, \quad L_{3}=-0.0044, \quad L_{4}=-1.289
$$

and since, the first and second exponents are positive, the system is hyperchaotic.

The Lyapunov dimension is a measure of system chaotic behavior degree, it can be defined according to Kaplan-York [29] conjecture and as follows:

$$
D_{k}=J+\frac{\sum_{i=1}^{J} L_{i}}{\left|L_{J+1}\right|}
$$

where $\mathrm{J}$ should be selected such that $\sum_{i=1}^{J} L_{i}>0$ and $\sum_{i=1}^{J+1} L_{i}<0$.

Then, using the Lyapunov exponents found for $a=0.05$ and $b=3.5$, the Lyapunov dimension is:

$$
D_{k}=3+\frac{\sum_{i=1}^{3} L_{i}}{\left|L_{4}\right|}=3+\frac{L_{1}+L_{2}+L_{3}}{\left|L_{4}\right|}=3.231
$$

\subsection{System analysis using bifurcation diagram}

Bifurcation diagrams is a great tool for investigating system behaviours with the change of one or more than one of system parameters. It can be used also to determine some system features like the rout to chaotic and the existing of coexisting attractors which is an interesting feature of the systems. Firstly, the bifurcation diagram for $b$ parameter has been found by fixing a at 0.02 and changing $b$ from 0 to 14 with 0.02 as a step size. At each step, the system is simulated for $500 \mathrm{sec}$. with $\mathrm{IC}=\{0.1,0.1,0.1,0.1\}$ and the local maxima of $\mathrm{x}_{2}$ have been found. The first $150 \mathrm{secs}$ have been ignored to ensure reaching steady state behaviour and cancelling transient effects. Figure 7 shows the resultant plot which clearly shows the chaotic nature of the system for the same range of $b$ obtained previously by using Lyapunov exponents. Also, bifurcation diagram for parameter a change from 0 to 0.2 has been found with 0.0001 step size and $b$ fixed at 3.5 which are the same conditions used in Lyapunov exponents section. Figure 8 shows the resultant plot which clearly shows consistency between the results obtained in this plot and the results obtained from the plot obtained for Lyapunov exponents where the same ranges of chaotic and regular behaviour of system can be noted in the two plots.

It is known from literature that there are three main routes to chaos. The first one is period doubling in which the period of periodic motion of the system is doubled at a specific point of bifurcation parameter and then and at a specific value of bifurcation parameter the motion becomes chaotic. The second route is the intermittency chaos in which periodic motion is replaced by chaotic at a certain value of bifurcation parameter. The last route is quasi periodic to chaos in which the system motion changes from fixed to periodic and quasi periodic and lastly chaotic motion appear, this rout is rear in systems. From Figures 3 and 7, it can be concluded that our system shows unusual rout to chaos where reverse quasi periodic rout is the rout of our system to chaos. The system is chaotic for $b<8$, then quasi periodic motion appears and for $b>13$ the system exhibits periodic motion.

To show the capability of the proposed system to exhibit coexisting attractors, we generate bifurcation diagram with two different ICs in each step. The first IC was $[0.1,0.10 .1,0.1]$ and it is fixed in each step and the other IC is selected from ten randomly generated ICs, where for each IC the maxima of x2 is determined and compared with those obtained for fixed IC condition to find the one which has more deviation. For the two IC, the maxima of $\mathrm{x}_{2}$ have been found and plotted against the bifurcation parameter which was b. the bifurcation parameter has been changed in the range [0.5 14] with 0.02 as a step size and a 
has been fixed to 0.02. The maxima of $\mathrm{x}_{2}$ for each IC have been plotted two different colours: red and green. The resultant plot is shown by Figure 9. From this figure it is clear that the system exhibits coexisting attractors for several ranges of the bifurcation parameter. As example for $b=2.2$ and $a=0.02$ and for the ICs $I_{1}=\{0,-3,1,-1.5\}, I_{2}=\{-0.2,-2,1,-1\}$ the system exhibits two chaotic coexisting attractors, but for $I_{3}=\{0,0,1,1\}$ the system reach to stable spiral response after $400 \mathrm{~s}$ transient chaos. Table 1 lists some examples of these coexisting attractors and Figures 10-15 shows the phase portraits plots of the attractors.

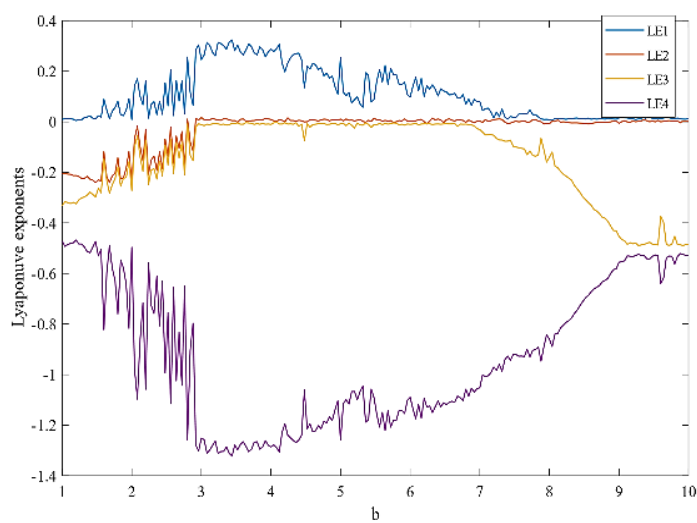

Figure 3. Lyapunov exponents with variation of $b$

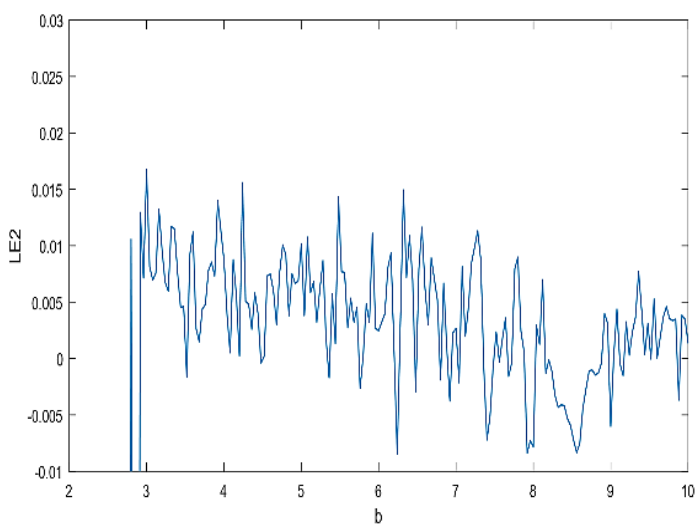

Figure 5. Second Lyapunov exponent versus b

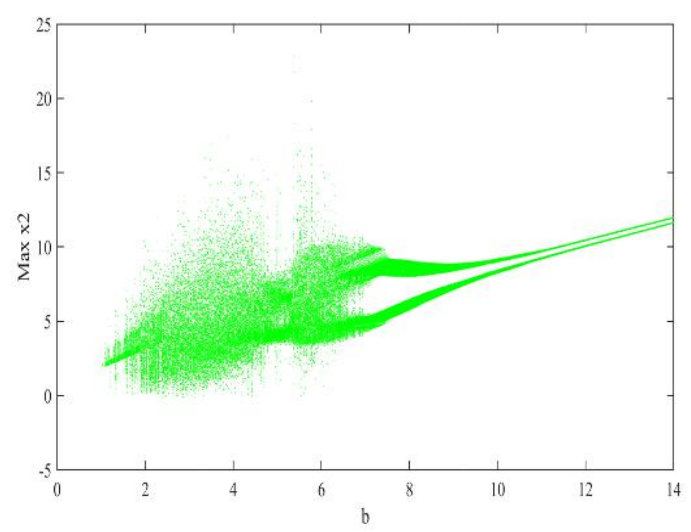

Figure 7. Bifurcation diagram for $b$

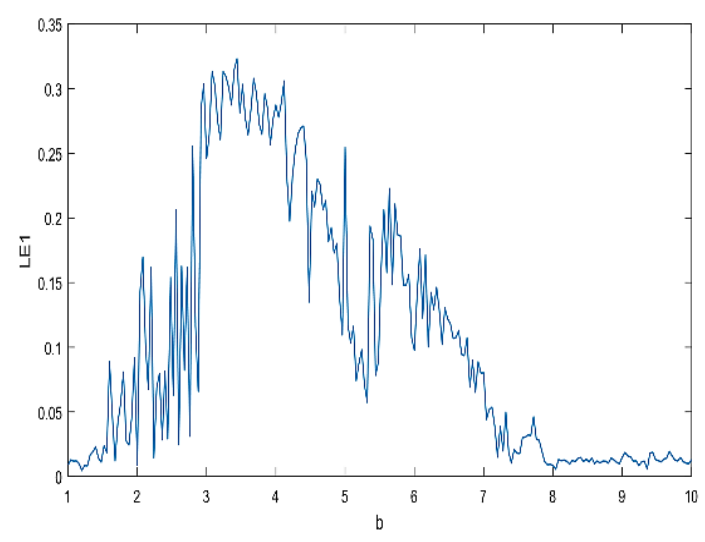

Figure 4. First Lyapunov exponent versus b

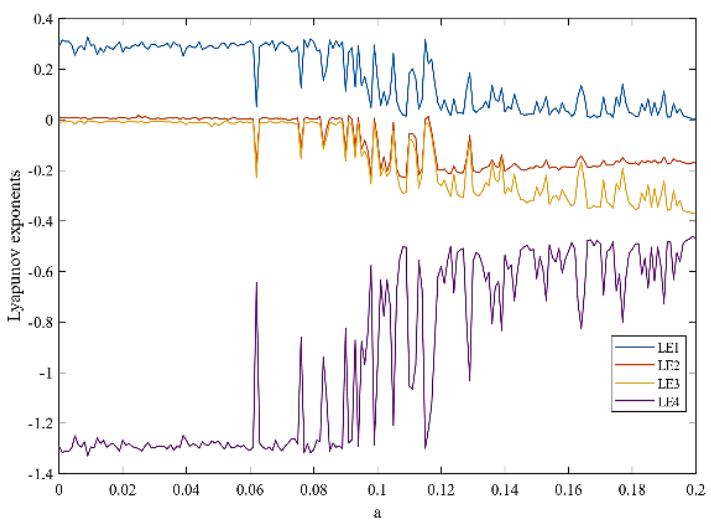

Figure 6. Lyapunov exponents with variation of a

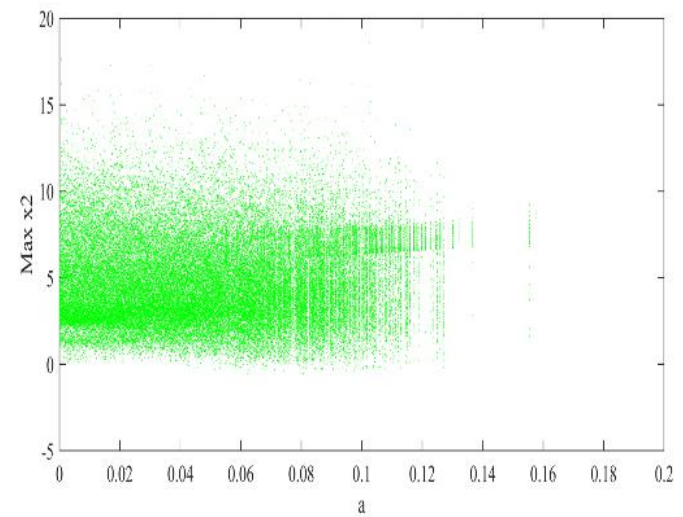

Figure 8. Bifurcation diagram for a 


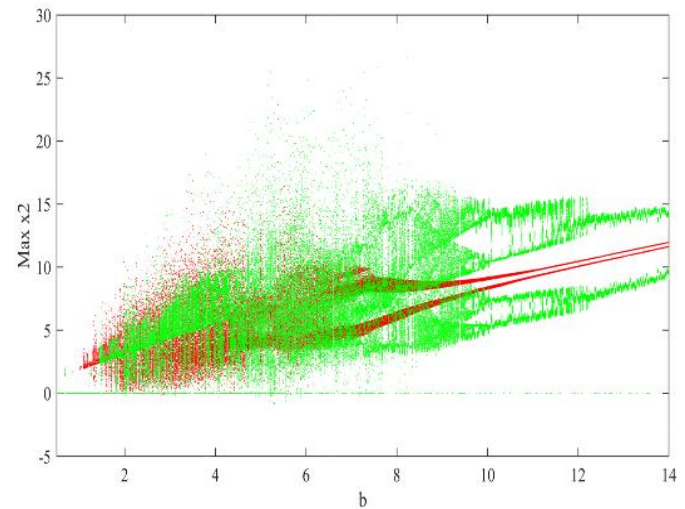

Figure 9. Bifurcation diagram for $\mathrm{b}$ with two ICs

Table 1. Some examples of the coexisting attractors

\begin{tabular}{ccc}
\hline b value & ICs & Behavior \\
\hline 2.2 & $\{0 .-3,1,-1.5\},\{-0.1,0,1,1\},\{-0.2,-2,1,-0.5\}$ & Three chaotic coexisting attractors. \\
2.2 & $\{0.1 .-3,1,-1.5\},\{2,0,1,1\},\{4,-2,3,-1\}$ & Three coexisting fixed stable points after transient chaos. \\
3.5 & $\{1,1,1,-1\},\{2,0,1,-1\},\{0,0,1-1\}$ & Three chaotic coexisting attractors. \\
5 & $\{0.6,-2,1,-1.5\},\{2,0,1,1\},\{-1,-2,3,-1\}$ & Three chaotic coexisting attractors. \\
8 & $\{0,-3,1,1.5\},\{0,0,1,1\},\{-0.2,-2,3,-1\}$ & Three quasi periodic coexisting attractors. \\
12 & $\{0,-3,1,-1.5\},\{0,0,1,1\},\{0,-2,3,-1\}$ & Three periodic coexisting attractors. \\
\hline
\end{tabular}

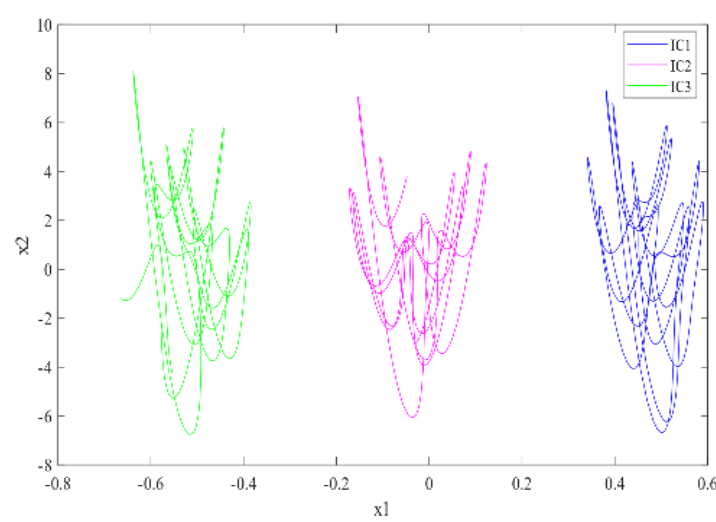

Figure 10. $b=2.2$ and $a=0.02$

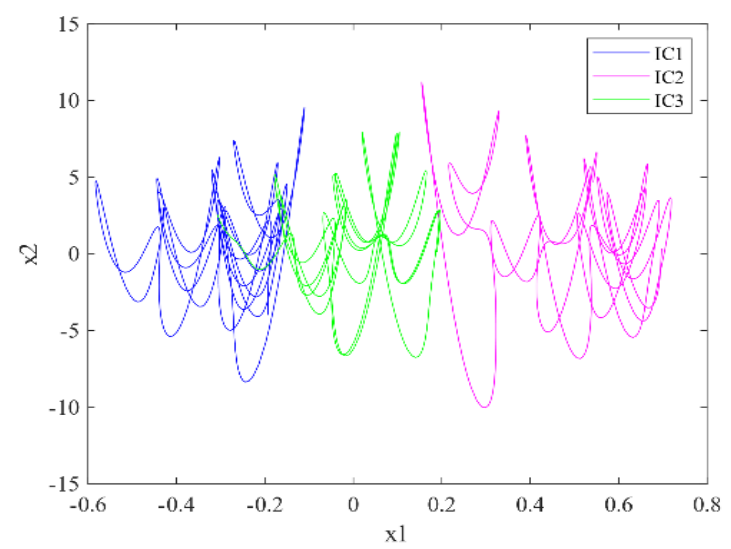

Figure 12. $b=3.5$ and $a=0.02$

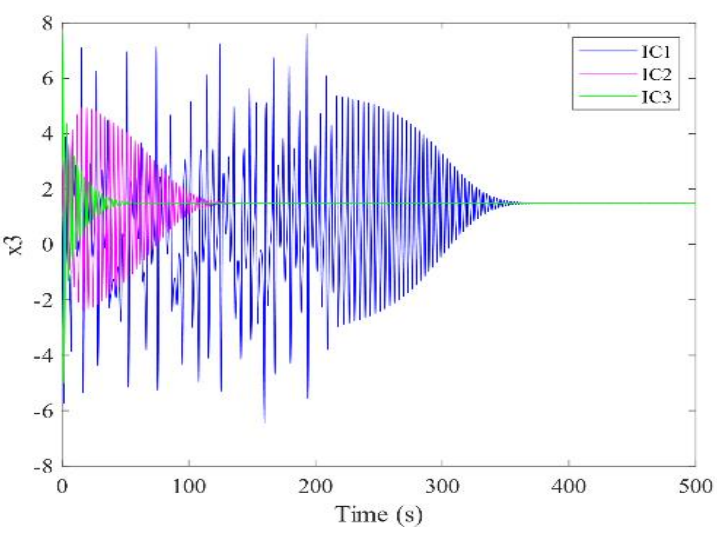

Figure 11. $b=2.2$ and $a=0.02$

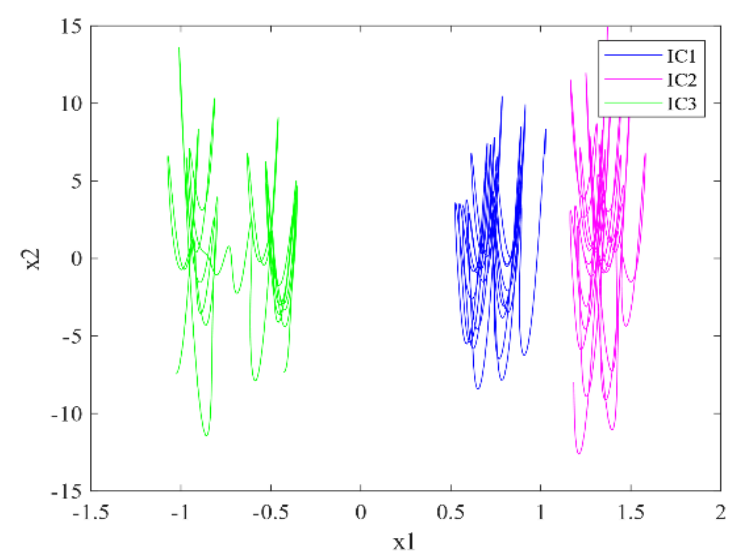

Figure $13 . \mathrm{b}=5$ and $\mathrm{a}=0.02$ 


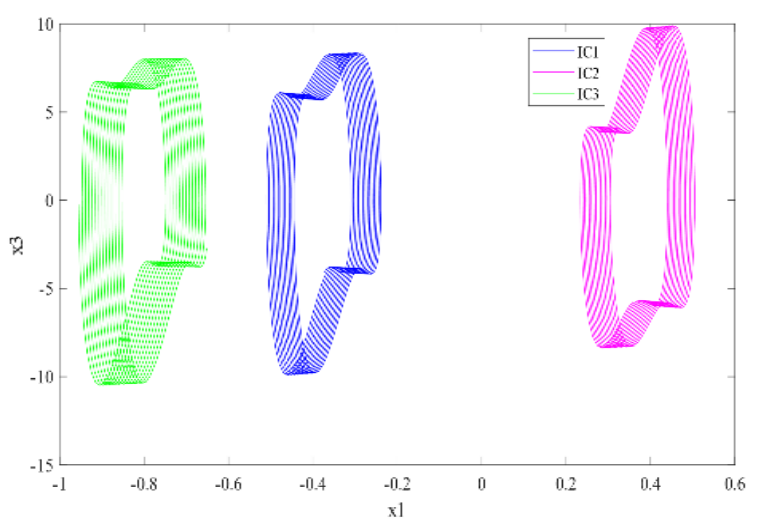

Figure $14 . \mathrm{b}=8$ and $\mathrm{a}=0.02$

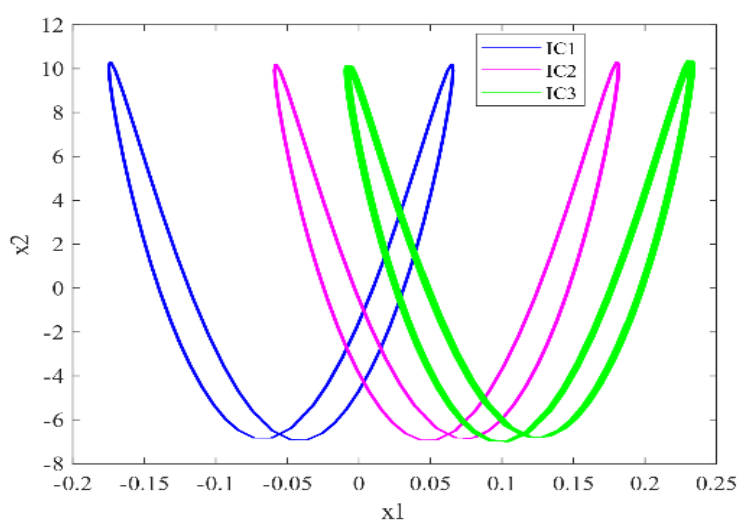

Figure $15 . \mathrm{b}=12$ and $\mathrm{a}=0.02$

\section{SYNCHRONIZATION CONTROLLER DESIGN}

In this section, a controller to synchronize two identical systems of the proposed system is designed. The system (1) is uncontrolled system or the master and its states output should be tracked or synchronized by the second system which is often called the slave. The slave system is the same as system (1) added to it the control signals $U=\left\{u_{1}, u_{2}, u_{3}, u_{4}\right\}$. Master system is represented by system 1 with a and $\mathrm{b}$ assumed to be unknown, while the slave system is represented by the following system (4):

$$
\begin{aligned}
& \dot{y}_{1}=a y_{3}+u_{1} \\
& \dot{y}_{2}=y_{3} y_{4}-b+u_{2} \\
& \dot{y}_{2}=y_{1}-y_{2} y_{4}+u_{3} \\
& \dot{y}_{4}=y_{3}-y_{4}+u_{4}
\end{aligned}
$$

The synchronization error is defined as (5):

$$
e_{i}=y_{i}-x_{i}, i=1,2,3,4
$$

Using this relation with (1), (4) and $\dot{e}_{l}=\dot{y}_{l}-\dot{x}_{l}, i=1,2,3,4$, the following system of equations is obtained:

$$
\begin{aligned}
& \dot{e}_{1}=a e_{3}+u_{1} \\
& \dot{e}_{2}=y_{3} y_{4}-x_{3} x_{4}+u_{2} \\
& \dot{e}_{3}=e_{1}-y_{2} y_{4}-x_{2} x_{4}+u_{3} \\
& \dot{e}_{4}=e_{3}-e_{4}+u_{4}
\end{aligned}
$$

Our target in the design procedure is to satisfy the following stable error dynamics:

$$
\dot{e}_{\imath}+k_{i} e_{i}=0, \quad \mathrm{i}=1,2,3,4
$$

Combining (6) and (5) and solving for U, we obtain:

$$
\begin{aligned}
& u 1=-k_{1} e_{1}-a_{n} e_{3} \\
& u 2=-k_{2} e_{2}-y_{3} y_{4}+x_{3} x_{4} \\
& u 3=-k_{3} e_{3}-e_{1}+y_{2} y_{4}-x_{2} x_{4} \\
& u 4=-k_{4} e_{4}-e_{3}+e_{4}
\end{aligned}
$$

Where $a_{n}$ and $b_{n}$ are the estimates of a and b. Substituting (8) into (7), yields:

$$
\begin{aligned}
& \dot{e}_{1}=-k_{1} e_{1}+a_{e} e_{3} \\
& \dot{e}_{2}=-k_{2} e_{2} \\
& \dot{e}_{3}=-k_{3} e_{3} \\
& \dot{e}_{4}=-k_{4} e_{4}
\end{aligned}
$$

where $a_{e}$ the error between the real parameter values of a and the estimate values $a_{n}$. 
The parameter estimation will be designed by Lyapunov theory. The following function is selected as a Lyapunov function candidate:

$$
V(t)=1 / 2\left(e_{1}^{2}+e_{2}^{2}+e_{3}^{2}+e_{4}^{2}+a_{e}^{2}\right)
$$

Then,

$$
\dot{V}(t)=e_{1} \dot{e_{1}}+e_{2} \dot{e_{2}}+e_{3} \dot{e_{3}}+e_{4} \dot{e_{4}}-\dot{a}_{n} a_{e}
$$

and,

$$
\dot{V}(t)=-k_{1} e_{1}-k_{2} e_{2}-k_{3} e_{3}-k_{4} e_{4}+a_{e}\left(e_{1} e_{3}-\dot{a}_{n}\right)
$$

If we put:

$$
\dot{a}_{n}=e_{1} e_{3}
$$

Then,

$$
\dot{V}(t)=-k_{1} e_{1}-k_{2} e_{2}-k_{3} e_{3}-k_{4} e_{4}
$$

Which is a negative semidefinite and the prove of system stability is completed.

\section{SYSTEM IMPLEMENTATION}

The proposed system has been implemented using microcontroller platform. We propose implementing of the system using the so-called Arduino MCU board, where Arduino uno board has been used to implement the system. Arduino boards are easy to use MCUs development boards. The used board is the Arduino uno which has $32 \mathrm{kB}$ flash memory for storing the program and $2 \mathrm{kB}$ of sram memory for storing program data and $1 \mathrm{kB}$ EEPOM which can be used for storing static data of the program. These specifications can cover all the requirements of implementing our system. The implementation program uses fourth order Runge-Kuta method to solve the ODE system of equations of our system with step size equal to $0.01 \mathrm{sec}$. For the purpose of testing the system, we have used Matlab to plot the phase portraits of the system states plane where the MCU board and laptop have been connected via serial communication. Figure 16 and Figure 17 shows the phase portrait of $\mathrm{x}_{1}, \mathrm{x}_{2}$ and $\mathrm{x}_{2}, \mathrm{x}_{3}$ planes received from MCU board for 100 secs. Run time with $\mathrm{a}=0.1$ and $\mathrm{b}=4$.

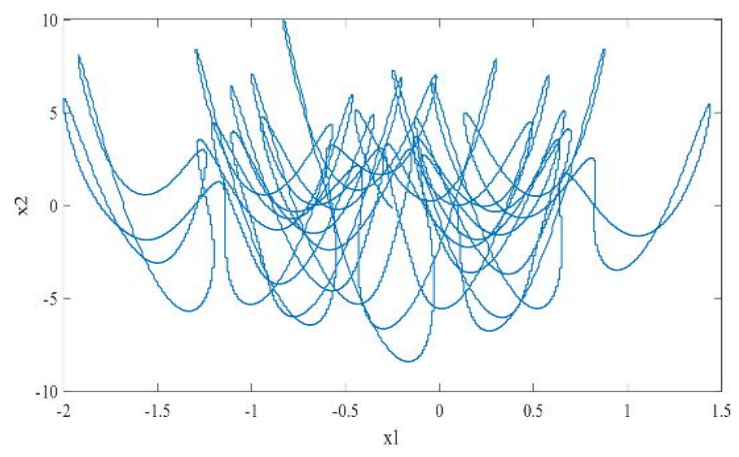

Figure 16. $\mathrm{x}_{1}, \mathrm{x}_{2}$ phase portrait of MCU system

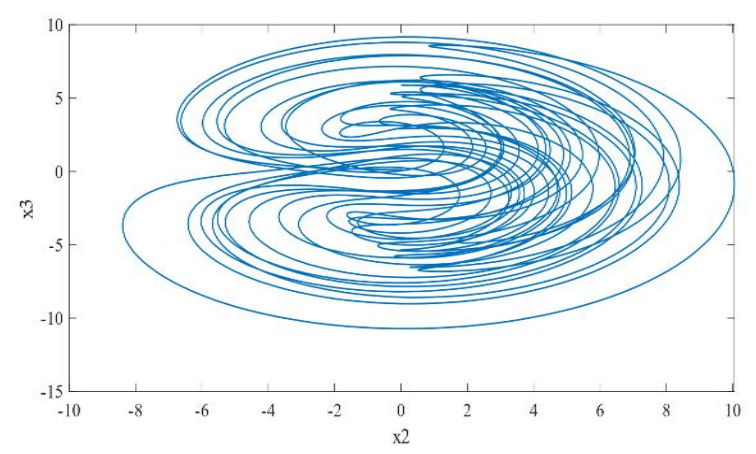

Figure 17. $\mathrm{x}_{2}, \mathrm{x}_{3}$ phase portrait of MCU system

\section{SIMULATION AND EXPERIMENTAL STUDY}

In this section, the theoretical results of synchronization of the proposed hyperchaotic system are investigated by simulation and experimentally. Also, a secure communication using our synchronization system is introduced and tested. As mentioned earlier, MATLAB 2018a script is used to write the simulation programs. The experimental study relayed on the system implemented on the MCU Arduino board and as described in the previous section. 


\subsection{Synchronization system}

The designed synchronization system is tested by using simulation and experimentally by using the MCU system as a master and the slave system is implemented on computer side using MATLAB with the following initial conditions for master and slave respectively:

$$
\begin{aligned}
& X=\left\{x_{1}, x_{2}, x_{3}, x_{4}\right\}=\{1,2,3,1\} \\
& Y=\left\{y_{1}, y_{2}, y_{3}, y_{4}\right\}=\{2,1,1,2\}
\end{aligned}
$$

The synchronization errors for simulation are shown in Figures 18 and 19 shows the experimental results. These figures show very excellent synchronization error with low tracking time and also show matching between the results obtained from simulation and experimentally.

\subsection{Secure communication application}

In this subsection, an application of chaotic system synchronization is presented. In analog secure communication using synchronized chaotic systems, the transmitted signal (sig) is added to one of the master system states (say $x_{1}$ ) to form the scripted signal (tr) to be transmitted to the receiver side with other signal required by slave to synchronize its output with master system. At the receiver side, the scripted (tr) signal is subtracted from the counterpart state of the slave ( $y_{l}$ in our case) to form the decrypted signal (rs), then:

At the transmitter:

$$
t r=\operatorname{sig}+x_{1}
$$

and at the receiver:

$$
r s=t r-y_{1}
$$

It is clear that if full synchronization is achieved, signal is completely retrieved, i.e. $r s=s i g$.

In our test, we used the implemented system on MCU as a transmitter side and the laptop as a receiver side. A sinusoidal signal $(\operatorname{sig}=2 \sin (20 t))$ is selected as a signal to be transmuted. Figure 20 shows the transmitted (sig) signal and the received (rs) signal. From this figure, it can be noted that the transmitted signal is tracked at the receiver with no error and in very short time.

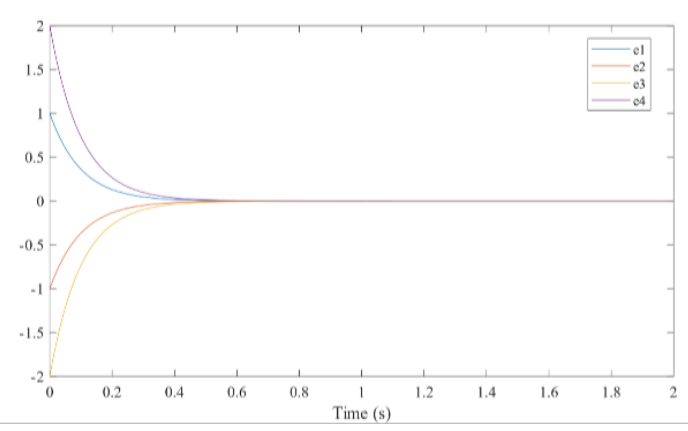

Figure 18. Synchronization errors for simulated system

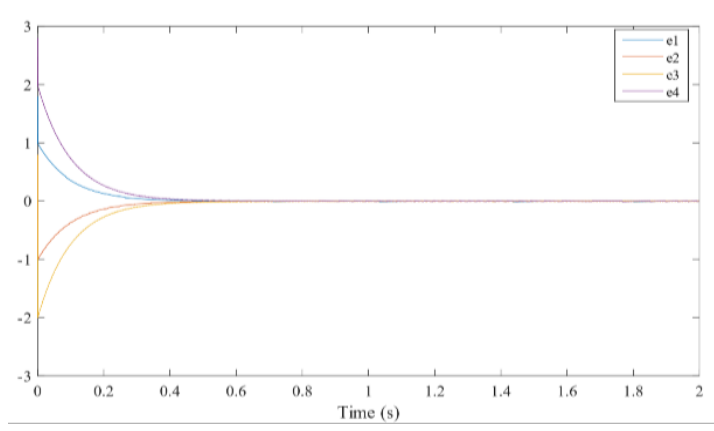

Figure 19. Synchronization errors for experimental system

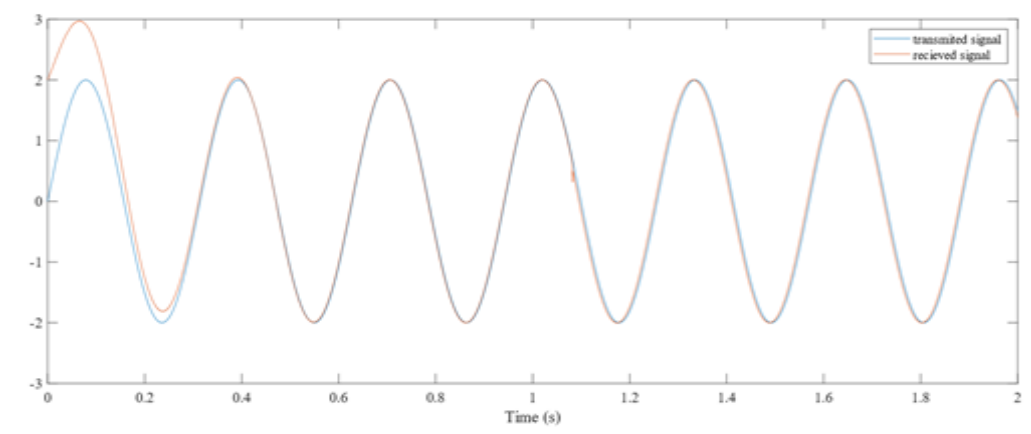

Figure 20. Transmitted and received signals 


\section{CONCLUSION}

In this paper, a new 4-dimensional hidden attractor hyperchaotic system has been proposed. Dynamical properties of the system have been investigated which includes coexisting feature of the system. After that, a synchronization system for the introduced system has been designed assuming unknown system parameters. The design procedure depended on simple controller with Lyapunov theory for the purpose of deriving update lows for the unknown parameters. An efficient and cost-effective implementation basing on Arduino boards shows very efficient implementation tool for chaos implementation and application where it has been used to build secure communication system. Simulation and experimental study have showed the effectiveness of the designed synchronization system and also the effectiveness of the secure communication system which can be designed basing on the designed synchronization system.

\section{REFERENCES}

[1] Alhadji Abba, et al., "Analysis, Circuit Design and Synchronization of a New Hyperchaotic System with Three Quadratic Nonlinearities, 2018, doi: 10.1007/978-3-319-71243-7_11.

[2] Mahmoud, Emad and Abood, Fatimah, "A novel sort of adaptive complex synchronizations of two indistinguishable chaotic complex nonlinear models with uncertain parameters and its applications in secure communications," Results in Physics, vol. 7, pp. 4174-4182, 2017, doi: 10.1016/j.rinp.2017.07.050.

[3] Liu, Yongjian and Yang, Qigui and Pang, Guoping, "A hyperchaotic system from the Rabinovich system," J. Computational Applied Mathematics, pp. 101-113, 2010, doi: 10.1016/j.cam.2009.12.008.

[4] Mahmoud, Emad, "Generation and suppression of a new hyperchaotic nonlinear model with complex variables. Applied Mathematical Modelling, vol. 38, no. 17-18, pp. 4445-4459, 2014, doi: 10.1016/j.apm.2014.02.025.

[5] A.Y. Aguilar-Bustos, and C. Cruz-Hernández, "Synchronization of discrete-time hyperchaotic systems: An application in communications," Chaos, Solitons and Fractals, 2009, doi: 10.1016/j.chaos.2008.05.012.

[6] Teerawat Sangpet, and Suwat Kuntanapreeda, "Finite-time synchronization of hyperchaotic systems based on feedback passivation," Chaos, Solitons and Fractals, vol. 132, pp. 1-10, 2020.

[7] Yousefpour, Amin, at al., "A fractional-order hyper-chaotic economic system with transient chaos," Chaos Solitons \& Fractals, vol. 130, pp. 1-12, 2019, doi: 10.1016/j.chaos.2019.109400.

[8] Faradja, Philippe and Qi, Guoyuan, "Analysis of multistability, hidden chaos and transient chaos in brushless DC motor," Chaos Solitons and Fractals, vol. 132, 2020, doi: 10.1016/j.chaos.2020.109606.

[9] Cao, Yanli, "Chaotic synchronization based on fractional order calculus financial system," Chaos, Solitons \& Fractals, vol. 130, pp. 1-7, 2020, doi: 10.1016/j.chaos.2019.109410.

[10] Khorashadizadeh, Saeed and Majidi, Mohammad-Hassan, "Chaos synchronization using the Fourier series expansion with application to secure communications," AEU-International Journal of Electronics and Communications, vol. 82, pp. 37-44, 2017, doi: 10.1016/j.aeue.2017.07.032.

[11] Al-Suhail, Ghaida and Tahir, Fadhil and Abd, Mariam and Pham, Viet-Thanh and Fortuna, Luigi, "Modelling of Long-Wave Chaotic Radar System for Anti-Stealth Applications," Communications in Nonlinear Science and Numerical Simulation, vol. 57, pp. 80-96, 2017, doi: 10.1016/j.cnsns.2017.09.008.

[12] Gleick J., "Chaos: making a new science," Open Road Media, 2011.

[13] Duta, Maitreyee, Binoy, Krishna and Roy, "A new fractional-order system displaying coexisting multiwing attractors; its synchronisation and circuit simulation," Chaos Solitons and Fractals, vol. 130, 2020, doi: 10.1016/j.chaos.2019.109414.

[14] Lorenz, E.N., "Deterministic nonperiodic flow," J. Atmos. Phys. vol. 20, pp. 131-141, 1963.

[15] Mahmoud, Emad., "Dynamics and synchronization of new hyperchaotic complex Lorenz system," Mathematical and Computer Modelling, vol. 55, pp. 1951-1962, 2012, doi: 10.1016/j.mcm.2011.11.053.

[16] A. T. Azar and S. Vaidyanathan, "Advances in Chaos Theory and Intelligent Control," Studies in Fuzziness and Soft Computing, Springer, Germany, vol. 337, 2016.

[17] Ababneh, Mohammad., "A new four-dimensional chaotic attractor," Ain Shams Engineering Journal, vol. 9, 2017, doi: 10.1016/j.asej.2016.08.020.

[18] Averbeck N., and Raines B., "Distributional chaos in dendritic and circular Julia sets," J. Math Anal Appl., vol. 428, no. 2, pp. 951-958, 2015.

[19] Alvarez G., Montoya F., Romera M., Pastor G., "Breaking parameter modulated chaotic secure communication system," Chaos Solitons \& Fractals, vol. 21, no. 4, pp. 783-787, 2004.

[20] Leonov G.A., Kuznetsov N.V., Vagaitsev V.I., "Hidden attractor in smooth Chua sys- tems," Physica D., vol. 241, pp. 1482-14826, 2012.

[21] Wang, Mengjiao, et al., "Dynamics, synchronization and circuit implementation of a simple fractional-order chaotic system with hidden attractors," Chaos, Solitons \& Fractals, vol. 130, 2020, doi: 10.1016/j.chaos.2019.109406.

[22] S. Vaidyanathan, V.T. Pham, C. Volos and A. Sambas, "A novel 4-D hyperchaotic Rikitake dynamo system with hidden attractor, itsproperties, synchronization and circuit design," Studies in Systems,Decision and Control, vol. 133, pp. 345-364, 2018.

[23] Pecora, L.M. and Carroll, T.L., "Synchronization in chaotic systems," Phys. Rev. Lett., vol. 64, pp. 821-824, 1990.

[24] Yang, T., "A survey of chaotic secure communication systems," Internat. J. Computational Cognition, vol. 2, no. 2, pp 81-130, 2004. 
[25] Arabyani, Homayoon and Saberi-Nik, Hassan., "Synchronisation of the hyperchaotic complex Lorenz system in a finite time," International Journal of Modelling, Identification and Control, vol. 25, no. 138, 2016, doi: 10.1504/IJMIC.2016.075273.

[26] A. Sambas, S. Vaidyanathan, S. Zhang, Y. Zeng, M. A. Mohamed and M. Mamat, "A New Double-Wing Chaotic System With Coexisting Attractors and Line Equilibrium: Bifurcation Analysis and Electronic Circuit Simulation," IEEE Access, vol. 7, pp. 115454-115462, 2019, doi: 10.1109/ACCESS.2019.2933456.

[27] Vaidyanathan, S., et al., "A new two-scroll chaotic system with two nonlinearities: Dynamical analysis and circuit simulation," TELKOMNIKA Telecommunication, computing, electronic and control, vol. 17, no. 5, pp. 2465-2474, 2019, doi: 10.12928/TELKOMNIKA.v17i5.10650.

[28] Wolf A, Swift J, Swinney H, Vastano J., "Determing Lyapunov exponents from a time series," Phys Lett B, vol. 737, no. 7, pp. 285-317, 1985.

[29] P. Frederickson, J. L. Kaplan, E.D. Yorke, J.A. Yorke, “The Liapunov dimension of strange attractors," J. Differ. Equ., vol. 44, pp. 185-207, 1983. 